\title{
Enquête
}

Archives de la revue Enquête

5 | 1989

Biographie et cycle de vie

\section{Biographies, flux, trajectoires}

Questions de l'extérieur. Avant-propos

Jean-Claude Passeron

\section{OpenEdition}

Journals

Édition électronique

URL : http://journals.openedition.org/enquete/77

DOI : 10.4000/enquete. 77

ISSN : 1953-809X

Éditeur :

Cercom, Éditions Parenthèses

Édition imprimée

Date de publication : 2 mars 1989

Référence électronique

Jean-Claude Passeron, «Biographies, flux, trajectoires », Enquête [En ligne], 5 | 1989, mis en ligne le 27 juin 2013, consulté le 30 avril 2019. URL : http://journals.openedition.org/enquete/77 ; DOI : 10.4000/ enquete.77

Ce document a été généré automatiquement le 30 avril 2019. 


\title{
Biographies, flux, trajectoires
}

\author{
Questions de l'extérieur. Avant-propos
}

\author{
Jean-Claude Passeron
}

1 Pour situer mon intervention, il faut accorder plus d'attention au sous-titre que je lui ai donné dans le programme, qu'à son titre : il s'agit bien de questions posées de l'extérieur, par un sociologue comme moi, qui n'ai jamais soumis directement à l'enquête des ensembles de données de type biographique (peut-être même l'ai-je évité) ni pratiqué assidûment les méthodologies qui leur sont liées. Je suis donc un intervenant exogène sur ce terrain, où a pris place, intensément depuis deux décennies, toute une grappe de travaux sociologiques et anthropologiques, dont les antécédents remontent cependant jusqu'au XIX ${ }^{e}$ siècle. Mais pour être posées de l'extérieur, mes questions n'en touchent pas moins, me semble-t-il, aux thèmes de débats que je vois se dessiner dans le programme.

Comme la ligne à laquelle je voudrais accrocher mon intervention est assez simple, je me permettrai de la caricaturer dans un slogan : il ne faut pas jeter le bébé de la struc ture avec l'eau du bain naturaliste. Je veux dire par là que le dépassement, actuellement observable dans de nombreuses recherches, des formes les plus mécaniques ou les plus abstraites d'un naturalisme déterministe constitue une avancée épistémologique; mais à la condition que l'on fasse mieux que ce que l'on dépasse, c'est-à-dire que l'on ajoute des contraintes à l'interprétation ; non que l'on régresse c'est-à-dire que l'on en soustraie. Or il me semble que, dans la conversion au travail biographique, l'avancée théorique s'accompagne assez fréquemment de la tentation de jeter par dessus bord tout ce qui, de près ou de loin, ressemble à une démarche de structuration des objets de recherche et à l'élaboration de méthodes visant à identifier des structures, qu'il s'agisse de structures synchroniques (cohérences de contemporanéité) ou de structures de séquences (segments de continuité ou figures de rupture).

\section{La chair et le squelette du temps}

3 On trouvera peut-être ambigu cet avant-propos. C'est que dans l'intérêt de plus en plus intense porté au «biographique», - entendant par là aussi bien le matériau 
autobiographique que tout matériau qui doit son organisation au temps de la vie des individus ou, aussi bien, au temps de l'enchaînement des générations - dans cet intérêt, dis-je, je trouve à la fois quelque chose de prometteur, et quelque chose de régressif. En tout cas, il ne manque pas de recherches qui présentent les aspects d'une régression infrasociologique. Prometteur et régressif, l'explication en est simple : c'est parce que la préoccupation du temps concret, événementiel, des successions individuelles ou collectives, des déroulements historiques particularisés, mêle deux sensibilités au temps, qui n'ont ni la même valeur théorique ni le même sens épistémologique. L'une, c'est la sensibilité perceptive à l'expressivité du concret, au réalisme littéraire du détail, à la singularité du devenir d'un individu ou d'une lignée. Et l'autre, c'est la sensibilité théorique à l'aspect longitudinal des phénomènes, à la structure des séquences de choix qui font, dès qu'elles ont quelque généralité, la structure d'un temps social ou d'une périodisation historique. Il se trouve que les deux sensibilités sont fortement associées ne serait-ce que parce que, psychologiquement, l'une engendre l'autre. Je voudrais seulement vous demander, assez naïvement à vous qui êtes, la plupart, spécialistes de ce champ d'étude, si vous ne trouvez pas que la première sensibilité théorique parasite la seconde, la fascine, y suscite avec insistance un risque de régression en-deçà d'acquis qui ont signalé le fondement des travaux sociologiques du xix siècle, puis ultérieurement des avancées comme celles des analyses fonctionnalistes ou des analyses structurales (acquis de généralisation et de comparaison). Je ne veux donc plaider ni la cause de la structure comme cause de l' "anti-humanisme théorique » (ainsi que l'énonçait Althusser dans les années soixante), ni la cause romanesque de la chair palpitante du concret, serrée d'aussi près que possible. Si l'on caricature l'une et l'autre de ces sensibilités narratives on définit deux pôles extrêmes de la description, qui, comme tous pôles extrêmes, attirent fortement les jeunes chercheurs en quête d'impressions fortes.

4 1. À un pôle, le récit biographique à l'état brut, rêvant d'exhaustivité, se berçant de la certitude que «rien n'est insignifiant». Ici ce qui est visé, épousé par le récit, ce qui procure l'impression de comprendre, c'est le fait de toucher du doigt, non seulement le "ceci s'est passé ainsi quelque part et pour quelqu'un ", mais de toucher du doigt la forme concrète, singulière, immédiatement compréhensible, de l'enchaînement des effets. Le récit biographique fasciné par ses propres pouvoirs de suggestion, c'est une visée utopique d'exhaustivité qui achète l'impression de compréhension de l'illusion d'immédiateté. À ce pôle donc, un risque majeur: l'évanouissement du problème théorique des traits pertinents de la description. Dès qu'il y a recherche du contact avec la réalité socio-historique dans ses aspects biographiques et individualisés apparait, en même temps que l'ambition la plus haute de la description anthropologique ${ }^{1}$, une illusion épistémologique qui suit cette ambition comme son ombre, l'illusion de la pertinence tous azimuts d'une expérience singulière. Puisque tout ça est du réel, du «direct», du singulier, que ce réel est touché du doigt, ramassé, raconté, récité, recueilli, filmé, il devient affectivement difficile d'en laisser perdre la moindre parcelle, chacune participant de la saveur totale du récit; il devient douloureux d'admettre que n'importe quel trait, n'importe quelle association de traits ne soit pas d'emblée pertinence.

5 On peut le dire autrement: tout semble pertinent parce que tout est senti comme métonymique. Dans le cas du récit biographique, la description, c'est-à-dire toutes les informations portées par la description doivent sans doute le surcroît de signification qu'elles revêtent pour l'auditeur à une habitude de lecteur contractée par chacun à la lecture du récit de fiction, où les « épaississements » de la narration, la constitution de 
tout détail en « détail synecdochique $»^{2}$ tendent à fonctionnaliser et donc à pertinentiser par rapport à l'intrigue toute information portant sur le contexte et vice versa... Bref, à ce pôle culmine un risque littéraire qui s'amplifie d'une illusion, en tant que telle liquidatrice du travail historique ou sociologique, l'illusion de la pan-pertinence du décrit : tout est pertinent, ce qui est dire, pour le dire comme l'empirisme logique, que le monde est indescriptible.

6 2. À l'autre pôle aussi peut se constituer un extrémisme qui, lui aussi, a eu ses militants : un style de description du devenir historique qui, glissant du structuralisme méthodologique au structuralisme dogmatique (du nominalisme au réalisme) ne veut plus connaître qu'une réalité, celle des traits pertinents et des systèmes de relations qui les constituent: réalisme des positions et des oppositions, des lois de transformation ou de reproduction, pour qui les devenirs individuels, cessant d'être des provocations à la description, ne peuvent plus être perçus que comme des "porteurs de la structure ", des occupants d'un système ou d'un champ, bref des agrégations de propriétés synchroniques. Ici les traits pertinents ne sont plus recherchés dans et par les fonctionnements biographiques (comme les traits pertinents d'un phonème le sont dans et par le fonctionnement phonétique d'une langue naturelle), ils sont retrouvés dans l'anonymisation statistique des porteurs du devenir comme de simples marqueurs d'une structure du devenir qui aurait pu se passer de biographies et donc, au sens strict, d'individus dotés d'identité. Pour autant que ce réalisme structural et/ou catégoriel concerne l'analyse longitudinale des phénomènes, on voit que les individualités biographiques s'y trouvent non pas stylisées - traitées, redécoupées, rapprochées ou opposées pour y construire le maximum de pertinence - mais oubliées, puisque dans ce style de description, les individus peuvent sans inconvénient méthodologique perdre leur identité à travers le temps. C'est ce qu'illustre, en toute recherche portant sur l'évolution au cours du temps d'un système de relations entre propriétés portées par des individus, l'avantage du « faux panel » sur le « panel vrai » : paradoxalement, il faut toujours conseiller (gain de temps, d'argent) pour cet objectif-là, la première solution méthodologique (qui fait porter les réitérations d'enquête à travers le temps sur des échantillons différents d'individus pourvu qu'ils soient caractérisés par les mêmes propriétés d'origine - celles dont on veut décrire, à travers le temps, l'évolution du lien qu'elles entretiennent avec d'autres propriétés -) dès lors que l'échantillonnage garantit au deuxième échantillon une représentativité dans la classe d'âge équivalente à celle du premier. La perte de la continuité biographique est ici sans conséquences.

7 On voit en quel sens j'appelle analyses de flux les analyses longitudinales qui ne connaissent les individus qu'en tant qu'ils peuvent se remplacer les uns les autres sous certaines conditions : elles procèdent comme ces analyses des courants marins, qui, pour leur propos, peuvent se satisfaire d'identifier dans des masses d'eau des caractéristiques de vitesse, de direction, de température, de composition chimique et d'en mesurer les variations sans s'astreindre au suivi individuel des molécules qui composent le courant et encore moins à la description de leur mouvement brownien. L'analyse des flux sociaux est une analyse des mouvements de propriétés dans une population, qui ne porte qu'en apparence sur les individus qui la composent, puisque les individus, identifiés par quelques traits pertinents ou un ensemble, fût-il très nombreux, de traits pertinents, restent pour l'analyse interchangeables dès lors que les mêmes traits pertinents peuvent être identifiés à l'occasion de coupes synchroniques successives, fût-ce dans une population différente. Il est évident que les usages classiques de l'instrument statistique 
tels qu'ils se sont stabilisés dans la sociologie quantitative favorisent la dérive vers une définition de l'analyse longitudinale, qui se réduit à l'analyse des flux catégoriels, c'est-àdire de l'évolution temporelle des co-occurences de propriétés.

Pour donner deux exemples qui situent ce qui est en jeu et ce qui est exclu par l'analyse des flux en cette forme objectiviste ou naturaliste, il faut reprendre ce que Godard objectait à la métaphore, utilisée par Bourdieu ${ }^{3}$, celle du plan du métro comme schéma contraignant des déplacements des voyageurs.

«Métaphore insuffisante», nous dit-il dans le programme du Colloque, "entre autres, parce que contrairement au réseau du métro qui n'évolue pas (ou très peu) les cursus sociaux institutionnalisés ont une histoire et sont en perpétuelle recomposition".

Certes, mais le problème n'est pas seulement d'histoire du réseau institutionnel dans lequel devraient voyager les biographies: Bourdieu, s'il rappelle souvent le préalable de la contrainte sociale, n'en suggère pas je crois, une cartographie fixiste; si le percement des tunnels relève d'une histoire trop lourde, le plan des villes fournira une meilleure approximation métaphorique (comme le suggérait Baudelaire un jour où il se trouvait en veine d'humeur sociologique : «Paris change plus vite, hélas, que le cœur des mortels »). Le problème du modelage des biographies n'est pas non plus seulement institutionnel, normatif ou représentationnel : il faut aussi penser à ces tracés que dessinent d'avance à chacun les chances sociales inscrites pour lui à chaque bifurcation de la chaîne de Markov en quoi se résout une biographie vue de l'extérieur, probabilités sociales qui obligent même quand elles ne sont revendiquées, utilisées ou explicitées nulle part et par personne : seule la sociologie quantitative a les moyens de les identifier et, par le filtrage des corrélations, de dégager et de dresser le plan, certes mobile historiquement, du réseau de contraintes par quoi les destins précèdent les biographies.

Le réductionnisme du schéma suggéré par la métaphore du "plan» ou de la «carte » tient, selon nous, à autre chose : ne connaissant que des itinéraires et des trajets individuels, il fait disparaître la considération des véhicules, c'est-à-dire des segmentations, plus ou moins contraignantes, plus ou moins durables, selon lesquelles sont conjoints ou disjoints les paquets d'individus véhiculés dans un réseau de déterminations. C'est pourquoi, je trouve très éclairante en sa simplicité la métaphore qu'utilisait Schumpeter dans son Essai sur les classes sociales ${ }^{4}$ : avant que Bourdieu ne descende dans le métro, Schumpeter avait en effet pris l'autobus. Les classes sociales (ou tous autres découpages attestés comme pertinents par une série d'effets), nous disait-il, sont comme des autobus dont le trajet est un objet spécifique de description, même si les véhicules ne contiennent plus à l'arrivée les mêmes voyageurs qu'au départ et, à la limite, au terminus plus aucun de ceux qui y étaient montés. Si peu marxiste qu'il fût, Schumpeter n'admettait pas que l'on pût liquider l'existence objective des classes et de leur histoire, par le constat qu'il y a de la mobilité sociale, c'est-à-dire que les individus ou les lignées entrent et sortent des classes sociales. L'étude de la mobilité sociale - intra- et inter-générationelle - constitue certes un champ de recherche (Schumpeter le traite longuement et comparativement), mais elle laisse ouvert le champ d'une autre description historique qui reste, en principe, indépendante de la première, celui du devenir des classes sociales dont la biographie collective ne se déduit pas des biographies individuelles. L'existence objective des classes (ou dirions-nous aujourd'hui moins aristotéliciennement, de la structure des relations qui les mettent en relations de conflit ou de coopération, de domination ou d'obéissance) 
possède des propriétés et une histoire qui ne se réduisent pas à celles des individus qui circulent entre elles, au cours d'une génération ou d'une génération à l'autre.

11 La circulation des autobus définit une événementialité qui s'inscrit dans la journée autrement que l'emploi du temps des voyageurs : l'autobus est arrivé ou a eu un accident, du retard ou non, etc. Il y a là un objet réel (relationnel, et certes pas substantiel), et non une hypostase métaphysique comme le répètent inlassablement les individualismes de conviction. La métaphore de l'autobus ne suggère pas de renoncer à l'analyse de la composition des flux mais elle tend à désindividualiser l'analyse de leur structure d'évolution ou de constance. Si vous opérez deux coupes synchroniques, au départ et à l'arrivée du bus, vous pouvez par exemple constater qu'au terminus il y avait moins de gens qu'au départ, qu'il y en avait plus ou qu'il y en avait autant, que le sex-ratio a changé ou la proportion de jeunes, etc., vous pouvez faire toutes sortes d'analyses comparatives entre les diverses stations, mais, même porteurs des mêmes propriétés, les voyageurs peuvent ne plus être du tout les mêmes: vous ne visez pas la même histoire, étant dans une autre méthodologie que celle qui permet de traiter les individus qui montent et qui descendent, et à qui vous auriez demandé leur biographie déambulatoire de la journée.

\section{Homunculus mundus}

L'intérêt des deux formes-limites que peut revêtir une appréhension descriptive du devenir est de dégager - la caricature a cette vertu - ce que fait spécifiquement chacun des deux choix méthodologiques : corrélat de ce qu'il ne peut pas faire. Si, précisément, l'analyse biographique éprouve plus de peine que l'analyse catégorielle à développer ses méthodes et ses astreintes propres, c'est que l'analyse d'une vie selon l'ordre biographique est toujours sous influence d'un modèle littéraire qui l'attend (au tournant), qui n'attend que sa rechute dans la littérature: derrière le récit de vie, le récit romanesque, derrière la construction du cas typique au travers des biographies, la recherche ou la comparaison des vies « exemplaires », qui vient d'encore plus loin dans l'histoire des genres littéraires.

Je crois que ce qui fait la force imaginaire de ces modèles littéraires et de quelques autres tient d'abord à une imagination philosophique, celle qui s'est enchantée, sous mille formes, de voir se refléter ou se miniaturiser le monde entier dans une vie d'homme, une lignée, une dynastie. Microcosmes répétant le macrocosme, ou harmonie préétablie entre monades-points de vue sur le monde, quelle économie de pensée que de savoir d'avance le monde, avec ses lois et ses structures, résumé en cette minuscule et émouvante singularité d'une vie individuelle : homunculus mundus! Une vie d'homme, c'est le « miroir du monde ", mieux le Monde même qu'il faudrait connaître en entier (comme Dieu le connaît ou, au moins, le Dieu-romancier) pour la comprendre vraiment, certitude qui, par conversion logique illégitime (mais combien fascinante) conduit au phantasme, paresseux mais intense, qu'il suffit de comprendre un homme pour avoir compris le monde. Chaque biographie contient Tout.

14 Lorsque cette nostalgie d'une connaissance qui réconcilierait le savoir du Grand Tout et le frisson d'authenticité que procure l'hic et nunc d'une individuation cesse d'être philosophique (bye bye Platon, Leibniz...), pour se faire anthropologique et se révèle capable de résister vertueusement à la gymnastique métonymique d'une vie trop bien racontée, le projet de narration biographique côtoie une dernière tentation, celle de la quête utopique d'un savoir intégral de l'individuel où l'encyclopédie des connaissances 
nomologiques viendrait se rassembler et se fondre dans la synthèse d'une connaissance clinique achevée: mobilisation et concentration dans la biographie, pour produire l'intelligibilité scientifique d'une vie d'homme, non plus du monde, mais de l'ensemble des savoirs produits par les sciences de l'homme. Je pense, bien sûr, à ce que laisse transparaître le projet interminable que devient L'idiot de la famille chez Sartre. Projet jamais terminé, et où ce qui nourrit l'interminabilité du projet d'éclaircir un trajet individuel (Flaubert devenant l'auteur de Madame Bovary), dans toutes ses dimensions, au sein des espaces de déterminations dans lesquels il s'inscrit, et au travers des relations dans lesquelles il entre, c'est l'existence des connaissances anthropologiques qui préexistent au projet de Sartre. Ce n'est pas Sartre qui fait sur Flaubert des découvertes sociologiques, historiques ou psychanalytiques; il faut au contraire que ces acquis de la recherche $\mathrm{du} \mathrm{xx}^{\mathrm{e}}$ siècle soient déjà là et déjà diffusés, déjà à portée de main pour que Sartre puisse entreprendre de faire valoir leur validité générale dans leur pertinence singulière pour la compréhension du cas Flaubert. Mais Flaubert comme M.X est inépuisable en tant qu'histoire survenue dans le monde. Loin de produire de quoi nourrir la curiosité de savoir, la biographie qui vise à l'explicitation, de l'individu en tant qu'individu, est condamnée à toujours exhiber de nouveaux détails en quête de leur raison d'être: nourrie à la nostalgie du savoir total, boulimique de toute connaissance, elle ne sera jamais rassasiée.

Nous voilà bien! semble-t-il, avec nos dilemmes méthodologiques et théoriques, à hésiter entre les excès d'un autobus qui aurait oublié ses voyageurs et les excès d'un biographe boulimique qui aurait oublié, pour l'amour de l'individu, ses devoirs comparatistes de sociologue. On voit en tout cas que l'hésitation porte sur les choix de traitement et non de matériau: le matériau biographique est du matériau historique comme un autre et souvent plus complet qu'un autre; le tout est de savoir qu'en faire. Comment produire, à partir d'un corpus de biographies, une intelligibilité longitudinale où l'on gagne quelque chose à être parti de biographies et non de mesures génériques ou synchroniques ? Si, dans mon propos, un danger paraît être balisé avec plus d'insistance que l'autre, c'est précisément parce que le récit biographique présente déjà en sa forme spontanée une organisation trop parlante qui paraît dispenser de tout travail de reconstruction puisqu'elle propose et impose, avant tout traitement, une structure d'interprétation. Elle en propose même plusieurs, ingénument lovées dans son évidence narrative préconstruite.

16 La première est sans aucun doute celle qui doit à son "évidence anté-prédicative " d'avoir en toute mythologie, dans les sagesses religieuses comme dans la sagesse des nations, fourni le fil conducteur le plus sollicité de l'intelligibilité des êtres et de leurs actes : le modèle génétique d'explication, avant d'avoir été plié aux règles de la méthode biologique ou clinique, a habité et hante encore sauvagement toute succession de phénomènes ou d'étapes de la narration. Post hoc propter hoc n'est pas, on le sait pourtant, un instrument probatoire de meilleur aloi dans la succession biographique que dans la succession historique. Si un récit bien mené le fait vite oublier, c'est que ce modèle premier doit sa puissance toujours renaissante à l'expérience immédiate que fait la conscience du suivi de ses propres décisions et, par-delà, à l'expérience primordiale, socialement et psychologiquement, de l'engendrement et de la filiation.

17 Ajoutons-y l'impression de comprendre en toute succession un développement sur le modèle de la croissance biologique: avant de se dire natura en latin, cet ordre de l'enchaînement des choses comme croissance et vie s'est d'abord dit phusis en grec, 
comme évidence que «le cheval engendre le cheval » et que la jeune plante ou le jeune animal grandissent et vieillissent. Dur chemin à remonter pour une méthodologie qui veut fonder autrement les intelligibilités de succession. Derrière le modèle biographique sommeille donc toujours un modèle génétique littéral, lui-même nourri de l'intelligibilité parente du modèle généalogique et, de fil en aiguille (ou plutôt : par une sémantique « en écheveau» pour parler comme Freud) des engendrements grandioses du modèle cosmogonique. Il n'y a donc pas que dans le conte ou l'épopée, dans les textes sacrés ou dans la politesse des cultures traditionnelles, que se dire "X fils d'Untel» suffit à s'expliciter et à s'expliquer comme homme et producteur d'actes ou comme dieu et maître d'un domaine de significations. Même la cosmogonie se glisse quotidiennement dans nos impressions de comprendre au fil de nos phrases d'historiens et de sociologues $\mathrm{du} \mathrm{Xx}{ }^{\mathrm{e}}$ siècle : dans l'Histoire du P.C. (bolchevique), sommet sublime de l'historiographie du marxisme d'État, les «déviations » semblent s'engendrer généalogiquement comme les héros de la « ligne juste » se transmettre le flambeau d'une génération à l'autre ; c'est, dira-t-on, que l'on est ici encore bien trop près de la représentation mythique de l'engendrement des «hérésies" les unes à partir des autres dans une histoire ecclésiastique, ou de celui des monstres dans une cosmogonie hésiodique ou babylonienne. Voire! Raymond Aron - excusez-moi, c'est l'exemple qui me vient suggérait avec insistance, dans son récit de Mai $68^{5}$ que les horrifiques événements et phantasmes de cette crise du symbolisme intellectuel devaient se comprendre comme un "retour», une remontée de l'imaginaire anarchiste, proudhonien et révolutionnaire français du XIX ${ }^{e}$ siècle : naissance d'enfants aux « dents agacées " par les fautes de leurs ancêtres et résurrection de grands ancêtres tutélaires ou maléfiques, se réfèrent à la même famille de modèles. Qui est sûr d'en être totalement exempt dans son interprétation des devenirs? Qui n'a jamais émargé à ce SMIG de l'évolutionnisme ? Tout récit, historique ou biographique, fourmille, si on y cherche cette petite bête-là, de questions résolues par le démon du récit avant même d'être posées. Qu'un savoir du devenir s'astreigne à ne jamais reposer sur la seule loi du récit est ici le seul exorcisme.

18 L'ordre biographique du récit appelle encore une autre forme de compréhension préréflexive qui ne contribue pas peu à en faire, pour le narrateur auto- ou hétérobiographique éprouvant fortement l'intuition confuse d'une nécessité faite homme, le plus court chemin capable d'en communiquer indivisiblement l'évidence concrète à un auditeur. Cette forme de compréhension repose sur l'impression que, pardelà et à travers l'inépuisable richesse singulière qui fait l'individuation d'un individu, la cohérence et l'unité de son existence relèvent d'une essence: un individu mérite d'autant plus d'être raconté qu'il incarne mieux ou plus exemplairement cette essence. Sans avoir l'air d'y toucher, la biographie se recommande ici de paraître servir sur un plateau la solution, aussi simple que l'œuf de Colomb, d'un problème qui se présente habituellement comme la quadrature d'un cercle logique, celui d'une synthèse opérée dans le langage entre les pouvoirs de la deixis (désignation en acte d'un être singulier à-nul-autre-pareil) et les pouvoirs de la " description définie » (définition opératoire par la possession d'une propriété ou d'une série de propriétés), bref, entre le discours par nom propre et le discours par nom commun : omne individuum ineffabile, on le sait sans avoir lu Aristote, mais c'est pourtant dans et par son individuation, en toute rigueur indicible ou interminable à dire, que l'on veut dire l'individu qu'on élit - et voilà qu'on se prend à croire, pour l'amour de ce projet, en une essence de la vie de Tel ou Telle, à croire que cette essence, si on la connaissait, permettrait de lire à livre ouvert toute la richesse de la 
vie dont elle est l'essence. Le biographe qui voudrait fonder le sens qu'il y a à chercher le sens d'une vie devrait s'avouer qu'il lui faut croire à quelque chose comme la Monadologie de Leibniz où la connaissance que possède Dieu des implications de chaque substance lui permet de lire tautologiquement dans la monade de César-bébé qu'il franchira le Rubicon.

Ce modèle essentialiste qui se nourrit de l'expérience originaire du récit de vie comme manifestation d'une valeur ou d'une idée, comme explicitation d'un sens, a donné force et forme commune à toutes sortes de traitements des vies célèbres, à travers les cultures populaires ou savantes, dans la légende ou l'histoire, en Occident comme en Orient, dans le De viris illustribus comme chez Sseu-ma Ts'ien. En petit dieu qu'il est le biographe sait seulement qu'il doit se résigner à ne ressaisir l'essence d'une vie qu'a posteriori, comme nécrologie en somme, et non a priori comme programme, ainsi qu'il est réservé au Grand Dieu informaticien de l'éternité logique selon Leibniz ou à son substitut le poète, libre programmeur de la Théogonie ou du Mahâbhârata. Reprenant la longue histoire intellectuelle de la Querelle des Anciens et des Modernes (qui s'instaure bien sûr dès l'Antiquité) et ponctuant les revivals un peu radoteurs de ce débat, Jauss a montré le rôle qu'ont joué en ces polémiques culturelles et littéraires les traitements comparatifs d'œuvres ou de vies d'auteurs montées en parallèle ${ }^{6}$. Si l'on remonte jusqu'à la forme paradigmatique du traitement des biographies, celle qu'ont illustré les Vies parallèles de Plutarque et que des admirations successives - sans oublier la traduction d'Amyot - ont relayée jusqu'à l'époque moderne, on aperçoit clairement l'essentialisme qui fait le ressort narratif de la comparaison biographique : de l'Antiquité au XVIII siècle en Europe, réfléchir sur des vies modèles, c'est toujours construire des portraits de vie que l'on fait concourir pour l'excellence de la manière dont ils réalisent une essence. Sauf, avec la méditation chrétienne, à mettre hors-concours une vie d'essence divine, ce qui simplifie la vie du biographe: Thomas a Kempis peut se reposer en toute spiritualité dans un doux tête-à-tête avec L'Imitation de Jésus-Christ.

Les Vies parallèles de Plutarque ne comparent qu'en apparence César et Alexandre ; la mise en parallèle des deux vies est une mise en raisonnement par le récit d'une question qui doit trancher de la remise d'un prix, la question de savoir quelle est de César ou d'Alexandre la vie qui incarne le mieux la figure du Grand guerrier, du Grand conquérant. Même chose pour Virgile et Homère, etc: l'essence est toujours en tiers entre les compétiteurs. La question n'a évidemment d'intérêt que si l'on suppose qu'il y a un «bien propre» (idion agathon) auquel tend chaque genre de vie, par exemple, pour reprendre une métaphore lieu commun chez les Grecs, qu'aux Jeux olympiques la vie contemplative (théorétique) des spectateurs a une autre fin, d'ailleurs supérieure à celle de la vie combattante (agonistique) des athlètes, laquelle à son tour se distingue et l'emporte sur la vie artisanale (banausique) des marchands de cacahuètes qui passent entre les gradins. Ne sous-estimons pas la force tranquille de cette structure, de cet essentialisme de fond qui a, dans nos traditions littéraires comme dans nos ornières argumentatives, marqué d'une évidence machinale l'idée qu'on ne peut raconter des vies qu'en les rapportant à un modèle de vie exemplaire.

21 En déplaçant un peu la notion d' « exemplaire ", on pourrait se persuader, à la lecture de certaines publications modernes, tant anthropologiques que sociologiques, que l'auteurchercheur, avant de choisir son héros tiré des profondeurs de l'anonymat populaire ou exotique, a mis en compétition des vies d'homme (ou aussi bien de village, de lignée, de famille étendue), non plus certes pour savoir quelle est celle qui mérite le prix d'excellence dans un genre de vie, mais au moins celle qui a remporté le pompon de « la 
plus typique ». En faisant concourir des vies, non plus pour l'excellence éthique, mais pour la typicité, pour la représentativité, on les fait en somme concourir - mais sans méthodologie ni règles du jeu-pour une excellence doxique, communicationnelle, l'excellence dans le pouvoir d'illustrer avec succès un schéma d'auteur - et dans le pire des cas un dada ou un stéréotype. Quand, sur cette base, le procédé sollicite suggestivement son lecteur, il a toutes les chances, accompagné d'un rien de caution scientifique, de déclencher à tous coups l'impression de comprendre en profondeur, de saisir la " vérité typique " (" plus vraie que la vérité statistique ») : pourvu qu'il vous soit détaillé sans trop de maladresse, un exemplaire sociographique (un profil de vie prélevé à la va-comme-je-te-pousse dans la réalité) vous fera toujours l'impression d'être un type sociologique, explicatif ou récurrent ${ }^{7}$. À voir pulluler les signes extérieurs de couleur locale prodigués en ces biographies, qui oserait douter de la typicité de ce paysan ou de cette brave fille, de cette famille ou de cette lignée, à qui un sociologue patenté, épaulé par un éditeur responsable, a consacré tout un livre?

Il ne faudrait cependant pas faire porter le chapeau de ce coup-là aux seuls sociologues. Dans un colloque récent l'historien Jacques Revel s'interrogeait perversement sur le caractère rarissime, chez ses collègues, d'une inquiétude au moins méthodologique, à propos du statut conféré dans la compréhension historique aux personnages et aux textes dont l'irruption se signale chez maint historien par un déictique qui revient comme un tic : « Voici Untel... » Le trope a de quoi inquiéter par sa fréquence. Que diable nous refile$\mathrm{t}$-on avec ce petit mot de présentation que sa légèreté fait passer comme une lettre à la poste ? « Voici X, bourgeois à Paris en 1700... »; « Voici Y, meunier de son état... »; « Voici Z, il lit Rousseau... »; "Voici une religieuse du Quesnoy, mûre, de quarante-cinq ans, mais, hélas! trop sensible... ». Ces beaux impromptus qui débarquent sans crier gare, généralement dans les moments cruciaux du récit ou de l'argumentation historique, ne sont pas des passants dont le passage est étranger au sens : ce sont eux qui donnent son allant, son nerf, sa couleur historienne au récit historique. Michelet, initiateur du grand style narratif dans l'Histoire du XIXe siècle, n'a pas négligé d'y accrocher quelques-uns de ses effets-chocs, par exemple l'apparition de l'huissier Maillard à l'Abbaye, au premier soir des massacres de septembre, pour y former son dérisoire et impressionnant tribunal :

« Il y avait au faubourg Saint-Antoine un personnage bizarre [...] un sombre et violent fanatique sous formes très froides, d'un courage et d'un sang-froid rares et singuliers $»^{8}$, celui qui, après la chute et la mort du premier assaillant, " passa le second, sans hésitation sans vertige » sur la planche, le fossé de la Bastille au 14 juillet et qu'on revit au 5 octobre « comme il faisait la conduite des femmes, ne permettant sur la route ni pillage ni désordre [...] Il avait près de 6 pieds ; sa taille, son habit noir, honnête, râpé et propre, sa figure solennelle, colossale, lugubre, imposaient à tous [...] Maillard ne reparait plus dans toute la Révolution : il resta comme enterré dans le sang. ?»

On voit bien ce qu'a fait Maillard dans la Révolution, moins clairement ce qu'il vient faire dans le texte de Michelet : y apparaît-il comme jacobin de base ? comme celui qui

" tenait également à deux choses : $1^{\circ}$ à ce que les aristocrates fussent tués $; 2^{\circ}$ à ce qu'ils fussent tués légalement, avec quelques formes, sur l'arrêt bien constaté du peuple, seul juge infaillible »?

Comme homme de sang à titre privé ? Modérateur des crimes inévitables? Envoyé de Danton? Idéal-type du ressentiment des bas fonctionnaires de justice? Figure énigmatique et donc littéraire ? On sent que Michelet n'en a cure, tout à son alchimie de la « résurrection » de Maillard. 
Mieux encore (ou pire), à y regarder de près, la présence de ces déictiques documentaires est capitale, non pas seulement dans l'économie stylistique du récit, mais dans l'économie de la preuve historique, puisque toujours gérés pour produire le choc du direct, ils renforcent et imposent la preuve en la théatralisant. Ces visiteurs inattendus de l'argumentation interviennent souvent après quelque suspense ménagé dans le texte par une question qui reste en balance ou une hypothèse en attente de sa preuve ${ }^{9}$. Petit coup de cymbale annonçant l'entrée en scène du texte qui tombe à pic, du témoignage improbable, de l'acte ou de l'acteur qui vient clore un débat ou contresigner une théorie, on a là de la preuve à l'état incandescent, de l'argument monté en épingle. Mais une fois admis le rôle de faire-valoir que joue dans la rhétorique historienne cette figure de l'Ecce homo, son sens probatoire n'en devient que plus ambigu. Simple illustration, dira-t-on; mais de quoi? Quels sont les traits assumés comme pertinents, par une telle exemplification, dans ce petit miracle de « résurrection » nous livrant intégralement son Lazare qui, du coup, ne manque jamais de faire effet d'époque dans son intégralité, pertinente ou non, représentative ou non? Reste évidemment qu'en hôte de ce festin aux convives improvisés, l'historien sera toujours plus excusable que le sociologue, puisqu'il doit lui prendre ce qu'il trouve c'est-à-dire ce qui reste ; s'il a la chance de mettre la main sur Monsieur X, chacun comprend qu'il est de son devoir professionnel de lui faire un sort. L'instruction d'une documentation biographique n'autorise pas une telle esquive au sociologue qui a, lui, le choix de ses dossiers.

\section{L'analyse des structures longitudinales : mots, concepts et tâches}

Enchaînons car, à trop vouloir exorciser de vieux démons, on pourrait croire que j'instruis un procès en sorcellerie, à tout le moins un procès d'intention, contre la méthode biographique - ce que je ne fais évidemment pas. Tout au contraire, dès qu'il y a mise en œuvre d'une méthode biographique, et non pas seulement d'un art du clin d'œil ou de la caresse littéraires (pour ne rien dire de la technique du direct à l'estomac), autrement dit, dès qu'il y a choix raisonné des critères régissant la constitution d'un corpus, associé à des règles d'analyse permettant conjointement l'identification de «traits pertinents » et le fonctionnement d'un protocole de traitement des données issu d'hypothèses portant sur la succession, la structure, le projet ou la mémoire biographiques, il me semble que tout est bon à prendre dans un échantillon de biographies. Un matériel biographique sera toujours plus complet, s'agissant de décrire les propriétés portées par un individu (ou, plus généralement, par une "unité statistique " quelle qu'elle soit), que le matériel recueilli par tout autre type de questionnement ou catégorisé selon toute autre grille d'observation, puisque les données de type biographique décrivent irremplaçablement, outre un riche ensemble de propriétés individuelles, l'ordre dans lequel ces propriétés sont advenues à l'individu et, à vrai dire, l'ont constitué, définissant ainsi par leur forme d'organisation le champ de toute interrogation sur le sens de cet ordre.

Les théories - et, partant, les méthodologies - qui visent à rendre compte de l'intelligibilité des ordres biographiques en les mettant en rapport avec des structures synchroniques ou diachroniques situées en amont ou en aval des devenirs individuels, sont aujourd'hui fort nombreuses, ainsi qu'en témoigne la diversité des mots-clés que l'on voit figurer dans le programme des interventions à ce colloque, comme on les voit aussi circuler, avec des acceptions point toujours semblables d'un auteur à l'autre, dans les 
travaux sociologiques : « âges de la vie », « cursus » ou « cycles de vie » en appellent-ils au même type de périodisation? "cheminement ", "parcours ", "trajet ", «tactique » ou « stratégie » posent-ils les mêmes questions à des données biographiques? «biographie ", « itinéraire ", « carrière ", « trajectoire » ont-ils le même statut théorique et conduisentils aux mêmes tâches de description et d'analyse ? Évidemment non, et pas seulement parce que certains de ces mots marquent l'image professionnelle des travaux qu'ils inspirent par la fonction de labelling qu'ils ont exercée au profit d'une école ou d'une œuvre. La métaphore ou l'analogie qu'ils portent ne désigne ni ne favorise la même conceptualisation, n'appelle l'attention sur les mêmes structures longitudinales. Mais tous, en principe, renvoient à un travail méthodique de reconstruction des données, par quoi la sociologie entend apporter sa réponse propre, irréductible à celle de la biographie littéraire, à une question qui peut toujours s'entendre en deux sens - que nous essayons ici de séparer - soit au sens existentiel qui conduit à l'exploration phénoménologique d'une expérience vécue, soit au sens de l'intelligibilité contrôlée que pratiquent les sciences sociales et qui seul nous intéresse, question qui dans les deux cas s'énonce cependant identiquement: qu'est-ce qui fait l'individuation d'une vie d'individu? On voit qu'aussitôt posée une telle question se démultiplie à volonté : une vie, comment ça se raconte? comment ça s'analyse ? est-ce que ça s'analyse comme ça se raconte? en quel sens peut-on parler de la structure d'une individuation ou de l'histoire d'un individu? est-ce chose pensable en théorie et empiriquement réalisable? quels rapports les structures qui rendent compte de la singularité d'un parcours individuel entretiennentelles avec l'impression d'un ordre choisi ou subi, ressentie comme évidence première par tout individu narrateur dans le récit de sa propre biographie ? Etc.

J'oublierai ici les nuances qui séparent les prédilections thématiques ou les subtilités du choix méthodologique pour discerner seulement deux versants de l'analyse sociologique des ordres biographiques. Si on laisse de côté la conception la plus abstraite de l'analyse des devenirs, celle que j'ai appelée " analyse catégorielle des flux », puisqu'elle néglige par principe l'individuation des biographies individuelles en les dissolvant dans l'anonymat agrégatif des biographies de catégories sociales, il me semble qu'il n'existe que deux cadres théoriques dans lesquels inscrire une recherche empirique sur les structures longitudinales de l'individuation: je dirais volontiers le premier cadre durkheimien puisqu'il se réfère à l'institutionnalisation du temps social, et le second sartrien (hé oui !) puisqu'il calque, sans l'apercevoir la plupart du temps, la structure du processus que décrivait Sartre dans sa phénoménologie du « pratico-inerte $»^{10}$.

\section{1. «Itinéraires » : l'inscription des parcours individuels dans la topographie et les calendriers institutionnels}

Les travaux qui prennent sens dans ce cadre, le plus classique assurément mais aussi le plus sûr puisqu'il balise un vaste champ propice à la découverte continue de nouveaux bornages et de nouveaux frayages sociaux, doivent leur cohérence théorique et leur ressort heuristique à un principe simple : le temps du devenir social des individus et des groupes est, avant toute possibilité de choix tactique ou stratégique, déjà structuré par des normes, des définitions sociales, des représentations ou, plus généralement encore, des chances objectives, socialement conditionnées, de déroulement ou d'orientation biographiques, "probabilités a posteriori », calculables au départ pour tout individu en fonction de ses appartenances, de longévité ou de scolarisation, de mariage comme de 
délinquance, etc., chances qui toutes peuvent d'ailleurs prendre, à chaque étape franchie, de nouvelles valeurs comme "probabilités conditionnelles». L'analyse longitudinale sait ici ce qu'elle doit chercher : la trace, plus ou moins marquée en toute biographie, des préstructurations plus ou moins cristallisées qui dessinent la géographie, historiquement mobile, dans laquelle les individus doivent nécessairement inscrire leurs parcours individuels. Élargissant encore le sens déjà très large que Durkheim donnait au concept d'« institution » dans Les Règles lorsqu'il y faisait entrer " toutes les croyances et tous les modes de conduite $»^{11}$ que l'individu trouve déjà « institués » avant d'avoir pu bouger le petit doigt, on pourrait dire que, à condition d'y comprendre aussi ces institutions en pointillé que dessinent tous les systèmes de restriction des choix significativement décelables, l'objet de cette sociologie du longitudinal n'est autre chose que l'institution biographique.

Tant qu'elle travaille dans ce cadre théorique, l'analyse sociologique n'a jamais affaire qu'à un registre de description et d'intelligibilité qu'elle connaît bien et dont elle maitrise facilement les méthodologies, puisque les concepts qui lui désignent ses tâches (représentations, normes, règles, attentes, institutions, chances sociales etc.) sont les mêmes que ceux qu'elle emploie depuis longtemps sur d'autres objets. On ne sousestimera cependant pas l'originalité des interprétations et le renouvellement de connaissance que permet ce style d'exploration de la socialité longitudinale : songeons par exemple à la portée théorique qu'ont eu, dans l'histoire des « mentalités », les travaux d'histoire sociale qui ont arraché la description de la scansion biographique à l'illusion du découpage «naturel », biologique, des « âges de la vie ». Il existe d'ailleurs des versions plus ou moins subtiles du schéma durkheimien d'une longitudinale précontrainte. On peut par exemple compliquer utilement la notion purement ordinale de l'ordre biographique en la complétant par le recours à la cardinalité qui enrichit mesures et comparaisons en permettant de construire et d'analyser un objet comme celui de rythme (avec ses retards ou précocités), associé à une biographie ou à une phase biographique. On peut aussi enrichir la description d'une structure longitudinale en prenant en compte les tâches de repérage qu'implique sa description comme graphe : les nœuds, les bifurcations où se redistribuent les chances d'emprunter sur la suite du parcours du graphe tel chemin plutôt que tel autre appellent alors une méthodologie spécifique mettant en œuvre des mesures et des critères opératoires.

31 On reste, je crois, dans une sociologie durkheimienne de l'exploration de l'amont des conduites tant que le groupe de transformations qui engendre les figures du parcours biographique peut se définir et se décrire comme "topographique». Le terme d'«itinéraire » pourrait, on le voit, être réservé à une telle conceptualisation des trajets biographiques puisqu'il symbolise assez adéquatement la manière dont un trajet se trouve dirigé par l'ensemble des déterminations inscrites en amont de chacune de ses avancées. Je songe évidemment à ce que furent, en tant que "guides de voyage " inscrivant les voyageurs dans un espace balisé, les itinéraires symboliques de la pensée ou de la liturgie religieuses, itinéraires de pèlerins au Moyen Âge ou, plus ritualisé encore, l'itinéraire de la Via Dolorosa à Jérusalem, admirablement décrit par Halbwachs en sa Topographie légendaire des Évangiles en Terre Sainte, comme une genèse laborieuse et complexe "encadrée » et animée par les mécanismes et les fonctions de la mémoire collective. 


\section{2. "Carrières " et «trajectoires " : la biographie à la fois en amont et en aval des structures longitudinales}

Les implications quelque peu naturalistes du schéma de l'« inscription institutionnelle " des biographies - ajoutées à la "vieille chanson » de la liberté humaine qui «berce d'âge en âge la misère » des sciences de l'homme - ont contribué à donner essor, sous des formes diverses, à un projet de description beaucoup plus ambitieux des rapports entre structures sociales et biographies individuelles. Toute la conjoncture scientifique favorise aujourd'hui dans les sciences sociales la remise en question d'un naturalisme intégral perçu comme « intégriste » (en l'occurrence on dit « réducteur »). De Weber et Pareto ou Simmel à l'«individualisme méthodologique " d'aujourd'hui, on n'a pas manqué de remarquer que les régularités de comportement qui paraissent s'imposer, en tant que contraintes sociales, comme des modelages préalables, apparaissent aussi (sauf à les hypostasier métaphysiquement), lorsqu'on prend la description dans l'autre sens, comme les produits, par agrégation, distillation, déposition, absorption, etc, d'actions qui, en tant qu'on essaie de les saisir empiriquement comme conduites, sont nécessairement des conduites d'individus: nul, disait Mannheim, n'a jamais rencontré dans la rue des «macranthropes» comme la Bourgeoisie, le Prolétariat ou une institution; Marx ou Durkheim auraient facilement avoué, à mon avis, qu'ils le savaient, mais n'entrons pas dans ce débat. Le fait est, si l'on écarte le coup d'éclat facile qui consiste à ne plus vouloir reconnaitre - y compris comme constructions théoriques - de structurations sociales qu'en aval et en tant que produits de décisions individuelles (intégrisme anti-holiste qu'adopte allègrement l'individualisme méthodologique contemporain), le fait est, dis-je, que le projet le plus recommandable (est-il le plus faisable, c'est une autre affaire) consiste à essayer de saisir la structuration des biographies à la fois comme un effet des structurations longitudinales qui se résument en amont dans l'«institution biographique » et comme le produit agrégé que l'action sociale des individus inscrit, en aval, dans le maintien ou la transformation de ces structures longitudinales.

Le choix n'est pas seulement de politique lexicale et on évitera même de le dire «dialectique ». Des outils de description comme le schéma interactionniste de la "carrière" ou celui de la «trajectoire" actualisant un habitus au travers des conjonctures qu'il traverse, montrent que le schéma sartrien de l'objectivation de la subjectivité et de la subjectivation de l'objectivité » définit un cheminement de la description praticable en sociologie.

Le concept de "carrière " permet ainsi, lorsque son utilisation tient ce que promet son contenu théorique, de saisir, par une description à la fois interprétative et explicative, le sens indissociablement subjectif et objectif que prend après coup comme carrière (pour le sociologue mais aussi sous le regard rétroactif du sujet) une succession d'actions, réactives, défensives, tactiques, anticipatrices etc., que celui-ci a choisies en son nom personnel pour gérer ses rapports avec le pouvoir contraignant d'un appareil qui lui a imposé anonymement la gradation prédéterminée des sanctions ou des récompenses correspondant à ses réponses (ou à ses abstentions) choisies. La " carrière délinquante " d'un sujet ou d'un groupe de sujets comparables, ou aussi bien une " carrière de malade ", font ainsi comprendre l'aspect indissociablement contraignant, non voulu (objectivé) et vécu comme personnel (subjective) d'une biographie, en les unifiant comme un processus "pratico-inerte» de confirmation réciproque des attentes de la biographie et de 
l'institution biographique. Ainsi, par exemple - cas perversement idéal- l'appareil policier et judiciaire confirme son fonctionnement en faisant confirmer ses attentes par les biographies qu'il traite, tout en confirmant les attentes subjectives qui unifient pour le délinquant le sens de l'appareil de contrainte dans l'unité inéluctable de sa carrière. Du moins en va-t-il ainsi lorsque l'analyse de carrière parvient à montrer, en chaque point déterminant de la carrière, le pas décisif accompli dans cette carrière comme le produit logiquement croisé d'une décision subjective (transaction, négociation, conflit, abstention) et de l'objectivité d'une contrainte de cheminement (cursus pré-établi dans une institution). Montrant comment le produit de la "praxis" d'un sujet lui revient comme une chose, dès qu'il est médiatisé par une chose sociale, le concept de «carrière » est une application de la phénoménologie du « pratico-inerte ».

Plus ambitieux encore, en ce qu'il est lié à une utilisation délicate de la notion d' " habitus » qui subsume théoriquement l'ensemble des structurations agissantes de l'amont et leur lien avec l'aval dans une structure de "reproduction», le concept de «trajectoire » a autorisé des interprétations inégalement riches et des pratiques de recherche inégalement contrôlées. À survoler quelques usages de ce concept, qui s'est beaucoup diffusé, on décèle au moins deux modèles d'intelligibilité à l'œuvre derrière les descriptions menées en termes de trajectoires. L'un - à la fois le plus joli et le plus discutable - tendrait à rendre "intégrale » l'explication du devenir biographique : ce n'est pas autre chose que le modèle mathématique de la courbe, tel du moins que le commentait didactiquement Leibniz quand il paraphrasait les bases du «calcul différentiel » en disant que la pente de la courbe est présente, par passage à la limite, en chaque point mathématique de la courbe. Il est arrivé à Bourdieu, indication métaphorique je pense, de se servir de ce souvenir. Mais devant le retour en force de Leibniz, autrement dit d'une pureté logique qui n'est autre que celle de la tautologie, le sociologue a le droit d'hésiter : qui croira qu'un individu soit chose si simple ou si docile qu'il puisse ainsi actualiser tout au long de sa trajectoire un habitus à lui inhérent, comme un point actualise tout au long de la courbe la fonction qui définit la courbe? Trop beau pour l'étoffe dont sont faites les choses sociales même devenues trajets : l'individu ne serait plus ici "porteur des structures » (hotte débordante de graphiques embrouillés), mais - fardeau plus léger, plus aérien, plus parfait - d'une simple équation. L'autre modèle, présent dans le terme même de «trajectoire ", est balistique et introduit en principe d'assez nombreuses exigences descriptives. On voit dès le principe qu'il s'agit de composer une force et une direction initiales propres à un mobile avec les champs de forces et d'inter-actions qu'il traverse: même dans le monde nomologique de l'astronautique, il est prudent de refaire plusieurs fois le calcul en cours de trajectoire. La balistique calcule dans un champ d'informations moins épuré que celui de la géométrie analytique ; que dire alors des champs de forces sociologiques?

On pourrait détailler d'autres schémas, mais dans cette famille de modèles, tous plus alléchants les uns que les autres, apparaissent deux difficultés nouvelles qui étaient épargnées aux schémas plus simples. L'une est méthodologique car la forme même des tâches de description et de mesure qu'ils imposent appelle la combinaison et le dosage d'approches fort hétérogènes entre elles: bonne occasion d'innover; danger aussi d'appeler «méthode» n'importe quelle embrouille ou la rechute dans les plaisirs de l'intuition. Ajoutons surtout un constat qui me semble valoir pour toute l'histoire des sciences sociales: plus un schéma théorique est subtil et ambitieux, plus s'accroît le risque, la montée de son prestige aidant, que son usage devienne machinal, c'est-à-dire 
verbal. Le bruit théorique lié à son emploi, l'espace idiomatique qu'il galvanise par sa seule apparition proposent trop de tentations de paresse. Ne rien dire à condition de le dire fort ou répétitivement: les mots de la théorie sociologique s'y prêtent plus que les mots de la théorie physique. Qui ne connait ces travaux d'imitateurs, plus soucieux des bénéfices de l'affiliation que des investissements du labeur, qui ne contiennent guère que quelque énumération ou quelque narration (certes fort bien gardées, par le jargon, de la tentation littéraire) et qui, hérissés de mots-tics comme celui de "carrière " mis à la sauce de n'importe quelle succession d'actes (délinquants, scolaires, amoureux, déambulatoires) ou comme celui d'" habitus de classe » qu'un malheureux individu semble condamné à transporter avec lui, comme un impedimentum, où qu'il aille, quoi qu'il fasse et sans qu'il en soit jamais fait d'autre inventaire que liturgique, n'ajoutent aucune information ou connaissance supplémentaire à celle qu'auraient communiquée l'énumération ou la narration en leur fome la plus plate, n'y ajoutant que la dissimulation de cette nudité informationnelle sous l'uniforme des grands galas théoriques.

Arrêtons là, pour ne pas faire long, l'entrée dans les questions que pose l'analyse, biographique ou non, du matériau biographique, dont je répète que je ne l'ai guère pratiquée. Comme tout le monde, j'ai fait des entretiens biographiques, mais je ne sais trop pourquoi, peut-être par prudence, j'ai toujours eu tendance à n'en faire qu'un usage illustratif ou anonymisé, y cherchant seulement la preuve de périodisations collectives ou la simple attestation statistique d'associations de traits. Bref, j'avoue que j'ai traité des entretiens biographiques comme s'ils n'étaient pas biographiques. Au fond, je n'ai jamais fait que de l'« analyse de flux » ou, parfois, de l'« analyse d'itinéraires ». C'est aussi, il faut le dire, que j'ai vu trop de chercheurs dire et ressasser pompeusement "carrière ", " trajectoire » ou « stratégie ", alors qu'à se référer aux matériaux ou aux méthodes qu'ils utilisaient et, en tout cas, aux résultats qu'ils obtenaient, ils auraient suffisamment rendu compte de ce qu'ils mettaient dans leur objet en le caractérisant banalement comme «succession ». Et voilà pourquoi mes questions sont des "questions de l'extérieur ", questions de la main gauche, posées par quelqu'un qui regarde avec curiosité vos travaux et qui vous encourage de la voix, comme on fait en pareil cas du bord de la touche en criant : « Macte animo, generose puer... Allez-y, allez-y! ». Mais regardez où vous mettez les pieds.

\section{NOTES}

1. Celle que Mauss formulait sous le label de «fait social total » comme «l'étude du concret, qui est du complet ", l'opposant ainsi aussi bien à l'abstraction psychologique qu'à l'abstraction sociologique : «le donné, c'est [...] le Mélanésien de telle ou telle île, et non pas la prière ou le droit en soi » (M. Mauss, "Essai sur le don», Sociologie et anthropologie, Paris, Presses universitaires de France, 1973, p. 276).

2. R. Jakobson, Essais de linguistique générale, Paris, Minuit, 1963, p. 63-66, où est analysée l'«affinité profonde qui lie le réalisme à la métonymie». Cf. sur ce point, J.-C. Passeron, «L'illusion de représentativité. Note sur un effet de littérature réaliste, conjointe à une remarque sur -graphie, -logie et -nomie », Enquête. Cahiers du CERCOM, 4, juin 1988. 
3. P. Bourdieu, "L'illusion biographique", Actes de la Recherche en Sciences sociales, 62-63, juin 1986.

4. J. Schumpeter, "Les classes sociales en milieu ethnique homogène ", in Impérialisme et classes sociales, Paris, Minuit, 1972, p. 183.

5. R. Aron, La révolution introuvable. Réflexions sur les événements de mai, Paris, Calmann-Lévy, 1968.

6. H. R. Jauss, Pour une esthétique de la réception, Paris, Gallimard, 1982, ch. III.

7. Cf. sur cette affirmation J.-C. Passeron, cité supra n. 2 (p. 105-112 $1^{\text {re }}$ éd.).

8. J. Michelet, Histoire de la Révolution française, tome IV, livre VII, ch. V.

9. Exemple classique, celui d'Architecture gothique et scholastique où l'introduction de l'« élément de preuve " confirmant la construction conceptuelle de l'ouvrage est longuement différée, jusqu'au dernier paragraphe de la dernière page: "On trouve dans l'Album de Villard de Honnecourt... ». Là seulement, comme bouquet final, est lancée au lecteur l'annotation idéaltypique, "Istud bresbiterium [...] inter se disputando », (E. Panofsky, Paris, Minuit, 1967, trad. fr., p. 130).

10. J.-P. Sartre, «De la "praxis" individuelle au pratico-inerte », Critique de la raison dialectique, Paris, NRF, 1960, p. 163-377.

11. E. Durkheim, "Deuxième préface", Les règles de la méthode sociologique, Paris, Presses universitaires de France, 1960, p. XII. 